\title{
The Structure of Uracil: A Laser Ablation Rotational Study
}

\author{
Vanesa Vaquero, M. Eugenia Sanz, Juan C. López and José L. Alonso \\ Grupo de Espectroscopía Molecular (GEM) \\ Departamento de Química Física y Química Inorgánica \\ Facultad de Ciencias, Universidad de Valladolid \\ 47005 Valladolid (Spain)
}

Supplementary Information 
Figure 1. A five $\mathrm{MHz}$ section of the $2_{2,0} \leftarrow 1_{1,1}$ rotational transition for the parent species of uracil, illustrating the complicated hyperfine structure ( $I, F$ labels are given). Because of the parallel arrangement of the molecular beam and the axis of the resonator each line appear as a Doppler doublet. The transition frequencies are calculated as the arithmetic mean of the Doppler components.

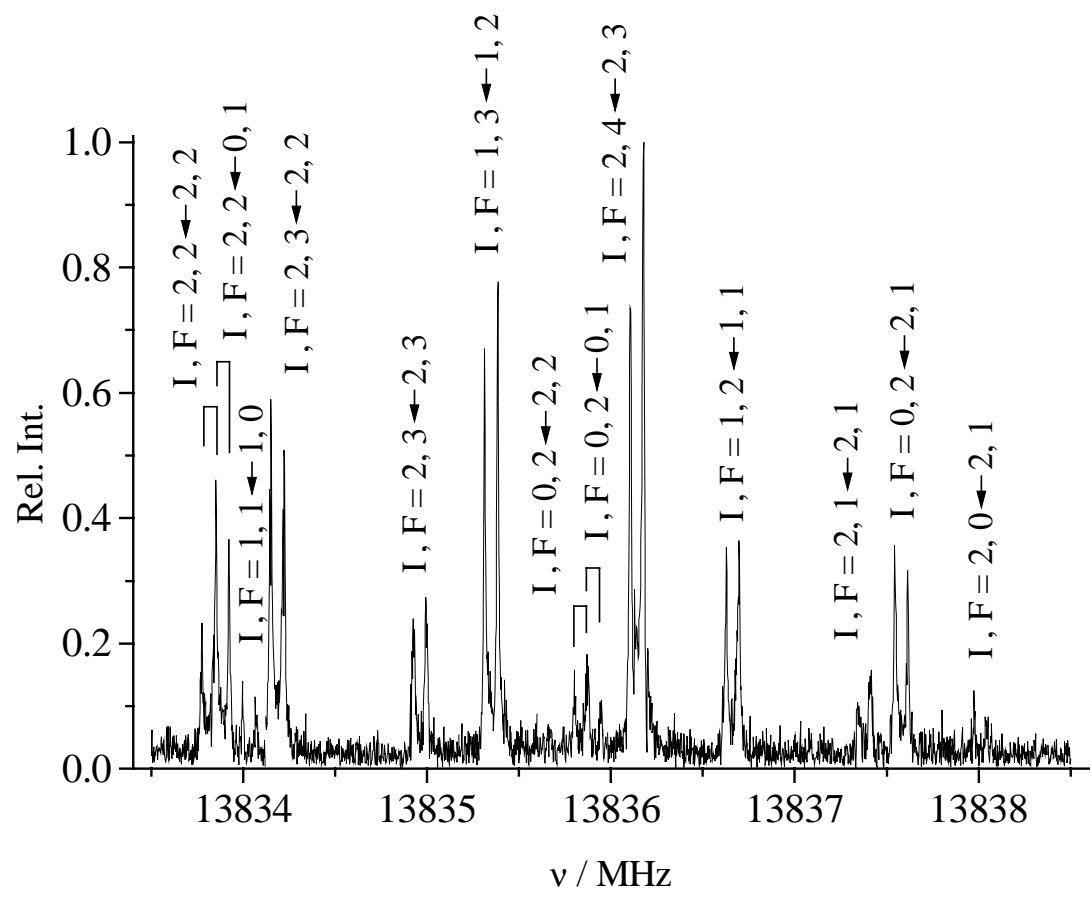


Table S1. Observed frequencies and residuals (in $\mathrm{MHz}$ ) for the nuclear quadrupole coupling hyperfine components of the parent uracil:

\begin{tabular}{|c|c|c|c|c|c|c|c|c|c|c|c|}
\hline$J^{\prime}$ & $K_{-1}^{\prime}$ & $K_{+1}^{\prime}$ & $J^{\prime \prime}$ & $K_{-1}^{\prime \prime}$ & $K_{+1}^{\prime \prime}$ & $I_{T}^{\prime}$ & $F^{\prime}$ & $I_{T}^{\prime \prime}$ & $F^{\prime \prime}$ & Obs / MHz & Obs-cal / MHz \\
\hline \multirow[t]{15}{*}{2} & 0 & 2 & 1 & 0 & 1 & 2 & 0 & 0 & 1 & 6547.227 & 0.002 \\
\hline & & & & & & 0 & 2 & 2 & 2 & 6547.592 & 0.005 \\
\hline & & & & & & 0 & 2 & 0 & 1 & 6547.672 & 0.002 \\
\hline & & & & & & 2 & 1 & 2 & 2 & 6547.790 & 0.001 \\
\hline & & & & & & 2 & 1 & 0 & 1 & 6547.872 & 0.000 \\
\hline & & & & & & 1 & 2 & 1 & 2 & 6548.344 & 0.001 \\
\hline & & & & & & 1 & 1 & 1 & 0 & 6548.809 & 0.001 \\
\hline & & & & & & 2 & 4 & 2 & 3 & 6548.899 & 0.003 \\
\hline & & & & & & 1 & 2 & 1 & 1 & 6548.899 & 0.005 \\
\hline & & & & & & 1 & 3 & 1 & 2 & 6549.178 & 0.003 \\
\hline & & & & & & 0 & 2 & 2 & 1 & 6549.444 & 0.004 \\
\hline & & & & & & 2 & 2 & 2 & 2 & 6549.569 & 0.006 \\
\hline & & & & & & 2 & 2 & 0 & 1 & 6549.648 & 0.003 \\
\hline & & & & & & 2 & 3 & 2 & 3 & 6550.007 & 0.002 \\
\hline & & & & & & 1 & 1 & 1 & 1 & 6550.192 & 0.004 \\
\hline \multirow[t]{12}{*}{2} & 1 & 2 & 1 & 1 & 1 & 0 & 2 & 2 & 2 & 6014.713 & 0.004 \\
\hline & & & & & & 0 & 2 & 0 & 1 & 6014.793 & 0.004 \\
\hline & & & & & & 2 & 1 & 0 & 1 & 6015.068 & -0.002 \\
\hline & & & & & & 1 & 2 & 1 & 2 & 6015.538 & 0.004 \\
\hline & & & & & & 2 & 0 & 2 & 1 & 6015.882 & 0.003 \\
\hline & & & & & & 1 & 2 & 1 & 1 & 6016.047 & 0.003 \\
\hline & & & & & & 2 & 4 & 2 & 3 & 6016.177 & 0.003 \\
\hline & & & & & & 0 & 2 & 2 & 1 & 6016.496 & 0.001 \\
\hline & & & & & & 1 & 3 & 1 & 2 & 6016.689 & 0.004 \\
\hline & & & & & & 2 & 3 & 2 & 2 & 6016.926 & 0.004 \\
\hline & & & & & & 2 & 2 & 0 & 1 & 6017.542 & 0.005 \\
\hline & & & & & & 2 & 3 & 2 & 3 & 6017.716 & -0.001 \\
\hline \multirow[t]{15}{*}{3} & 0 & 3 & 2 & 0 & 2 & 0 & 3 & 2 & 3 & 9470.774 & 0.001 \\
\hline & & & & & & 2 & 2 & 2 & 2 & 9471.034 & 0.000 \\
\hline & & & & & & 1 & 3 & 1 & 3 & 9471.451 & 0.001 \\
\hline & & & & & & 2 & 1 & 2 & 1 & 9471.759 & 0.002 \\
\hline & & & & & & 1 & 3 & 1 & 2 & 9472.283 & 0.000 \\
\hline & & & & & & 2 & 5 & 2 & 4 & 9472.299 & 0.002 \\
\hline & & & & & & 1 & 2 & 1 & 1 & 9472.565 & 0.001 \\
\hline & & & & & & 1 & 4 & 1 & 3 & 9472.620 & 0.003 \\
\hline & & & & & & 2 & 4 & 2 & 3 & 9472.649 & 0.002 \\
\hline & & & & & & 2 & 2 & 2 & 1 & 9472.811 & 0.004 \\
\hline & & & & & & 2 & 2 & 0 & 2 & 9473.008 & -0.001 \\
\hline & & & & & & 2 & 3 & 2 & 2 & 9473.026 & 0.002 \\
\hline & & & & & & 2 & 3 & 2 & 3 & 9473.412 & 0.002 \\
\hline & & & & & & 2 & 4 & 2 & 4 & 9473.755 & -0.001 \\
\hline & & & & & & 1 & 2 & 1 & 2 & 9473.860 & 0.001 \\
\hline \multirow[t]{9}{*}{3} & 1 & 3 & 2 & 1 & 2 & 0 & 3 & 2 & 3 & 8931.718 & 0.002 \\
\hline & & & & & & 2 & 2 & 2 & 2 & 8931.970 & 0.003 \\
\hline & & & & & & 1 & 3 & 1 & 3 & 8932.646 & 0.002 \\
\hline & & & & & & 2 & 1 & 2 & 1 & 8933.160 & 0.001 \\
\hline & & & & & & 2 & 5 & 2 & 4 & 8933.768 & 0.003 \\
\hline & & & & & & 1 & 3 & 1 & 2 & 8933.797 & 0.002 \\
\hline & & & & & & 1 & 2 & 1 & 1 & 8933.917 & 0.002 \\
\hline & & & & & & 0 & 3 & 0 & 2 & 8933.931 & 0.002 \\
\hline & & & & & & 2 & 4 & 2 & 3 & 8934.002 & 0.003 \\
\hline
\end{tabular}




\begin{tabular}{|c|c|c|c|c|c|c|c|c|c|c|c|}
\hline & & & & & & 1 & 4 & 1 & 3 & 8934.063 & 0.003 \\
\hline & & & & & & 2 & 3 & 2 & 2 & 8934.393 & 0.003 \\
\hline & & & & & & 2 & 2 & 2 & 1 & 8934.435 & 0.001 \\
\hline & & & & & & 2 & 2 & 0 & 2 & 8934.715 & -0.001 \\
\hline & & & & & & 2 & 3 & 2 & 3 & 8934.929 & 0.002 \\
\hline & & & & & & 2 & 4 & 2 & 4 & 8935.548 & 0.004 \\
\hline & & & & & & 1 & 2 & 1 & 2 & 8935.714 & 0.000 \\
\hline \multirow[t]{15}{*}{4} & 0 & 4 & 3 & 0 & 3 & 0 & 4 & 2 & 4 & 12158.347 & -0.001 \\
\hline & & & & & & 2 & 3 & 2 & 3 & 12158.589 & -0.002 \\
\hline & & & & & & 1 & 4 & 1 & 4 & 12159.018 & -0.003 \\
\hline & & & & & & 2 & 2 & 2 & 2 & 12159.197 & -0.004 \\
\hline & & & & & & 1 & 4 & 1 & 3 & 12160.187 & 0.000 \\
\hline & & & & & & 2 & 6 & 2 & 5 & 12160.187 & 0.000 \\
\hline & & & & & & 1 & 3 & 1 & 2 & 12160.455 & -0.001 \\
\hline & & & & & & 1 & 5 & 1 & 4 & 12160.483 & -0.001 \\
\hline & & & & & & 2 & 5 & 2 & 4 & 12160.491 & 0.000 \\
\hline & & & & & & 2 & 3 & 2 & 2 & 12160.580 & -0.001 \\
\hline & & & & & & 2 & 4 & 2 & 3 & 12160.816 & -0.001 \\
\hline & & & & & & 2 & 3 & 0 & 3 & 12161.227 & -0.001 \\
\hline & & & & & & 2 & 4 & 2 & 4 & 12161.581 & 0.001 \\
\hline & & & & & & 2 & 5 & 2 & 5 & 12161.947 & -0.004 \\
\hline & & & & & & 1 & 3 & 1 & 3 & 12162.031 & -0.001 \\
\hline \multirow[t]{14}{*}{4} & 1 & 4 & 3 & 1 & 3 & 1 & 4 & 1 & 4 & 11770.174 & -0.003 \\
\hline & & & & & & 2 & 2 & 2 & 2 & 11770.452 & -0.002 \\
\hline & & & & & & 2 & 6 & 2 & 5 & 11771.558 & 0.000 \\
\hline & & & & & & 1 & 4 & 1 & 3 & 11771.593 & 0.000 \\
\hline & & & & & & 0 & 4 & 0 & 3 & 11771.646 & -0.001 \\
\hline & & & & & & 1 & 3 & 1 & 2 & 11771.689 & -0.001 \\
\hline & & & & & & 2 & 5 & 2 & 4 & 11771.708 & 0.002 \\
\hline & & & & & & 1 & 5 & 1 & 4 & 11771.773 & 0.000 \\
\hline & & & & & & 2 & 3 & 2 & 2 & 11771.958 & 0.001 \\
\hline & & & & & & 2 & 4 & 2 & 3 & 11771.967 & 0.000 \\
\hline & & & & & & 2 & 3 & 0 & 3 & 11772.744 & -0.002 \\
\hline & & & & & & 2 & 4 & 2 & 4 & 11772.893 & 0.000 \\
\hline & & & & & & 2 & 5 & 2 & 5 & 11773.482 & -0.002 \\
\hline & & & & & & 1 & 3 & 1 & 3 & 11773.609 & 0.000 \\
\hline \multirow[t]{4}{*}{5} & 1 & 5 & 4 & 1 & 4 & 1 & 4 & 1 & 3 & 14539.594 & -0.005 \\
\hline & & & & & & 1 & 6 & 1 & 5 & 14539.648 & -0.003 \\
\hline & & & & & & 2 & 4 & 2 & 3 & 14539.758 & -0.004 \\
\hline & & & & & & 2 & 5 & 2 & 4 & 14539.789 & -0.004 \\
\hline \multirow[t]{9}{*}{1} & 1 & 1 & 0 & 0 & 0 & 2 & 1 & 2 & 2 & 5213.635 & 0.003 \\
\hline & & & & & & 2 & 1 & 0 & 0 & 5213.635 & 0.003 \\
\hline & & & & & & 1 & 1 & 1 & 1 & 5214.369 & 0.001 \\
\hline & & & & & & 2 & 3 & 2 & 2 & 5214.625 & 0.004 \\
\hline & & & & & & 1 & 2 & 1 & 1 & 5214.883 & 0.004 \\
\hline & & & & & & 0 & 1 & 2 & 2 & 5215.340 & 0.004 \\
\hline & & & & & & 0 & 1 & 0 & 0 & 5215.340 & 0.003 \\
\hline & & & & & & 2 & 2 & 2 & 2 & 5215.416 & 0.000 \\
\hline & & & & & & 1 & 0 & 1 & 1 & 5215.677 & 0.003 \\
\hline \multirow[t]{6}{*}{2} & 1 & 2 & 1 & 0 & 1 & 2 & 0 & 0 & 1 & 7874.293 & 0.003 \\
\hline & & & & & & 0 & 2 & 2 & 2 & 7874.824 & 0.002 \\
\hline & & & & & & 2 & 1 & 1 & 0 & 7874.832 & 0.004 \\
\hline & & & & & & 0 & 2 & 0 & 1 & 7874.907 & 0.002 \\
\hline & & & & & & 2 & 1 & 2 & 2 & 7875.104 & 0.000 \\
\hline & & & & & & 1 & 2 & 2 & 2 & 7875.112 & 0.004 \\
\hline
\end{tabular}




\begin{tabular}{|c|c|c|c|c|c|c|c|c|c|c|c|}
\hline & & & & & & 2 & 1 & 0 & 1 & 7875.187 & 0.000 \\
\hline & & & & & & 1 & 2 & 1 & 2 & 7875.665 & 0.003 \\
\hline & & & & & & 2 & 0 & 2 & 1 & 7876.064 & 0.003 \\
\hline & & & & & & 1 & 2 & 1 & 1 & 7876.216 & 0.003 \\
\hline & & & & & & 2 & 4 & 2 & 3 & 7876.326 & 0.006 \\
\hline & & & & & & 1 & 1 & 1 & 0 & 7876.636 & 0.005 \\
\hline & & & & & & 0 & 2 & 2 & 1 & 7876.679 & 0.003 \\
\hline & & & & & & 1 & 3 & 1 & 2 & 7876.817 & 0.004 \\
\hline & & & & & & 2 & 1 & 2 & 1 & 7876.956 & -0.001 \\
\hline & & & & & & 2 & 3 & 2 & 2 & 7877.038 & 0.003 \\
\hline & & & & & & 2 & 2 & 0 & 1 & 7877.657 & 0.003 \\
\hline & & & & & & 2 & 3 & 2 & 3 & 7877.865 & 0.001 \\
\hline & & & & & & 1 & 1 & 1 & 1 & 7878.015 & 0.004 \\
\hline & & & & & & 2 & 2 & 2 & 3 & 7878.403 & 0.002 \\
\hline 2 & 2 & 0 & 1 & 1 & 1 & 2 & 2 & 0 & 1 & 13833.906 & -0.002 \\
\hline & & & & & & 1 & 1 & 1 & 0 & 13834.049 & -0.002 \\
\hline & & & & & & 2 & 3 & 2 & 2 & 13834.217 & 0.002 \\
\hline & & & & & & 2 & 3 & 2 & 3 & 13835.010 & 0.000 \\
\hline & & & & & & 1 & 3 & 1 & 2 & 13835.310 & 0.001 \\
\hline & & & & & & 2 & 4 & 2 & 3 & 13836.121 & 0.001 \\
\hline & & & & & & 1 & 2 & 1 & 1 & 13836.652 & 0.002 \\
\hline & & & & & & 0 & 2 & 2 & 1 & 13837.590 & 0.001 \\
\hline 2 & 2 & 1 & 1 & 1 & 0 & 1 & 2 & 2 & 1 & 12980.633 & -0.002 \\
\hline & & & & & & 2 & 1 & 2 & 1 & 12980.641 & 0.003 \\
\hline & & & & & & 0 & 2 & 2 & 1 & 12980.784 & 0.002 \\
\hline & & & & & & 2 & 0 & 2 & 1 & 12981.095 & -0.002 \\
\hline & & & & & & 1 & 1 & 1 & 1 & 12981.175 & -0.002 \\
\hline & & & & & & 2 & 2 & 2 & 3 & 12981.374 & 0.000 \\
\hline & & & & & & 2 & 3 & 2 & 3 & 12981.648 & 0.000 \\
\hline & & & & & & 1 & 2 & 1 & 1 & 12982.098 & 0.000 \\
\hline & & & & & & 2 & 4 & 2 & 3 & 12982.441 & 0.003 \\
\hline & & & & & & 1 & 3 & 1 & 2 & 12982.585 & 0.001 \\
\hline & & & & & & 2 & 2 & 0 & 1 & 12982.833 & 0.001 \\
\hline & & & & & & 2 & 2 & 2 & 2 & 12982.989 & -0.004 \\
\hline & & & & & & 1 & 2 & 1 & 2 & 12983.175 & 0.002 \\
\hline & & & & & & 2 & 3 & 2 & 2 & 12983.264 & -0.004 \\
\hline & & & & & & 1 & 1 & 1 & 0 & 12983.872 & 0.000 \\
\hline & & & & & & 1 & 2 & 0 & 1 & 12984.091 & -0.003 \\
\hline & & & & & & 2 & 1 & 0 & 1 & 12984.100 & 0.004 \\
\hline & & & & & & 0 & 2 & 0 & 1 & 12984.242 & 0.002 \\
\hline & & & & & & 2 & 1 & 2 & 2 & 12984.263 & 0.006 \\
\hline & & & & & & 0 & 2 & 2 & 2 & 12984.400 & -0.002 \\
\hline & & & & & & 2 & 0 & 0 & 1 & 12984.554 & -0.001 \\
\hline 3 & 1 & 3 & 2 & 0 & 2 & 2 & 1 & 2 & 2 & 10258.700 & -0.001 \\
\hline & & & & & & 0 & 3 & 2 & 3 & 10259.580 & 0.004 \\
\hline & & & & & & 2 & 2 & 2 & 2 & 10259.975 & 0.000 \\
\hline & & & & & & 1 & 3 & 1 & 3 & 10260.286 & 0.003 \\
\hline & & & & & & 2 & 1 & 2 & 1 & 10260.475 & 0.002 \\
\hline & & & & & & 1 & 3 & 1 & 2 & 10261.119 & 0.004 \\
\hline & & & & & & 1 & 4 & 2 & 3 & 10261.141 & -0.002 \\
\hline & & & & & & 2 & 5 & 2 & 4 & 10261.193 & 0.003 \\
\hline & & & & & & 1 & 4 & 1 & 3 & 10261.701 & 0.003 \\
\hline & & & & & & 1 & 2 & 1 & 1 & 10261.738 & 0.000 \\
\hline & & & & & & 2 & 4 & 2 & 3 & 10261.863 & 0.003 \\
\hline & & & & & & 2 & 3 & 2 & 2 & 10262.402 & 0.003 \\
\hline
\end{tabular}




\begin{tabular}{|c|c|c|c|c|c|c|c|c|c|c|c|}
\hline & & & & & & 2 & 3 & 2 & 3 & 10262.788 & 0.003 \\
\hline & & & & & & 2 & 4 & 2 & 4 & 10262.969 & 0.001 \\
\hline & & & & & & 1 & 2 & 1 & 2 & 10263.035 & 0.002 \\
\hline \multirow[t]{14}{*}{3} & 2 & 2 & 2 & 1 & 1 & 2 & 1 & 2 & 0 & 15643.509 & -0.003 \\
\hline & & & & & & 0 & 3 & 0 & 2 & 15643.796 & -0.006 \\
\hline & & & & & & 2 & 1 & 2 & 1 & 15643.932 & -0.008 \\
\hline & & & & & & 2 & 2 & 2 & 1 & 15643.932 & -0.008 \\
\hline & & & & & & 1 & 3 & 1 & 2 & 15643.953 & -0.007 \\
\hline & & & & & & 1 & 2 & 1 & 2 & 15643.963 & 0.002 \\
\hline & & & & & & 1 & 4 & 1 & 3 & 15644.499 & -0.004 \\
\hline & & & & & & 1 & 3 & 1 & 3 & 15644.499 & -0.004 \\
\hline & & & & & & 1 & 2 & 1 & 1 & 15644.832 & -0.006 \\
\hline & & & & & & 2 & 3 & 2 & 3 & 15644.897 & -0.005 \\
\hline & & & & & & 2 & 4 & 2 & 3 & 15644.897 & -0.005 \\
\hline & & & & & & 0 & 3 & 2 & 2 & 15644.897 & -0.005 \\
\hline & & & & & & 2 & 3 & 2 & 2 & 15645.149 & -0.006 \\
\hline & & & & & & 2 & 2 & 2 & 2 & 15645.149 & -0.007 \\
\hline \multirow[t]{16}{*}{3} & 0 & 3 & 2 & 1 & 2 & 0 & 3 & 2 & 3 & 8142.915 & 0.001 \\
\hline & & & & & & 2 & 2 & 2 & 2 & 8143.023 & -0.003 \\
\hline & & & & & & 2 & 2 & 2 & 3 & 8143.560 & -0.002 \\
\hline & & & & & & 1 & 3 & 1 & 3 & 8143.812 & 0.000 \\
\hline & & & & & & 2 & 1 & 2 & 1 & 8144.442 & 0.000 \\
\hline & & & & & & 1 & 2 & 1 & 1 & 8144.742 & 0.001 \\
\hline & & & & & & 2 & 4 & 2 & 3 & 8144.788 & 0.001 \\
\hline & & & & & & 2 & 5 & 2 & 4 & 8144.873 & 0.001 \\
\hline & & & & & & 1 & 4 & 1 & 3 & 8144.979 & 0.001 \\
\hline & & & & & & 0 & 3 & 0 & 2 & 8145.129 & 0.002 \\
\hline & & & & & & 2 & 1 & 2 & 0 & 8145.335 & -0.003 \\
\hline & & & & & & 2 & 2 & 2 & 1 & 8145.492 & -0.001 \\
\hline & & & & & & 2 & 3 & 2 & 3 & 8145.553 & 0.002 \\
\hline & & & & & & 2 & 2 & 0 & 2 & 8145.772 & -0.002 \\
\hline & & & & & & 2 & 4 & 2 & 4 & 8146.333 & 0.001 \\
\hline & & & & & & 1 & 2 & 1 & 2 & 8146.538 & -0.002 \\
\hline \multirow[t]{14}{*}{4} & 1 & 4 & 3 & 0 & 3 & 0 & 4 & 2 & 4 & 12558.574 & -0.002 \\
\hline & & & & & & 2 & 3 & 2 & 3 & 12558.912 & 0.000 \\
\hline & & & & & & 1 & 4 & 1 & 4 & 12559.257 & -0.002 \\
\hline & & & & & & 2 & 2 & 2 & 2 & 12559.392 & -0.003 \\
\hline & & & & & & 1 & 4 & 1 & 3 & 12560.426 & 0.000 \\
\hline & & & & & & 0 & 4 & 0 & 3 & 12560.450 & 0.001 \\
\hline & & & & & & 1 & 5 & 1 & 4 & 12560.853 & -0.001 \\
\hline & & & & & & 1 & 3 & 1 & 2 & 12560.865 & 0.001 \\
\hline & & & & & & 2 & 3 & 2 & 2 & 12560.898 & -0.003 \\
\hline & & & & & & 2 & 5 & 2 & 4 & 12560.918 & 0.000 \\
\hline & & & & & & 2 & 4 & 2 & 3 & 12561.344 & 0.001 \\
\hline & & & & & & 2 & 4 & 2 & 4 & 12562.106 & 0.000 \\
\hline & & & & & & 2 & 5 & 2 & 5 & 12562.376 & -0.002 \\
\hline & & & & & & 1 & 3 & 1 & 3 & 12562.442 & 0.001 \\
\hline \multirow[t]{8}{*}{4} & 0 & 4 & 3 & 1 & 3 & 0 & 4 & 2 & 4 & 11369.132 & -0.004 \\
\hline & & & & & & 2 & 3 & 2 & 3 & 11369.211 & -0.005 \\
\hline & & & & & & 1 & 4 & 1 & 4 & 11369.937 & -0.002 \\
\hline & & & & & & 2 & 2 & 2 & 2 & 11370.255 & -0.003 \\
\hline & & & & & & 2 & 5 & 2 & 4 & 11371.275 & -0.003 \\
\hline & & & & & & 2 & 6 & 2 & 5 & 11371.293 & -0.001 \\
\hline & & & & & & 1 & 5 & 1 & 4 & 11371.401 & -0.002 \\
\hline & & & & & & 0 & 4 & 0 & 3 & 11371.417 & -0.002 \\
\hline
\end{tabular}




$\begin{array}{llllll}2 & 4 & 2 & 3 & 11371.438 & -0.003 \\ 2 & 2 & 2 & 1 & 11371.531 & -0.003 \\ 2 & 3 & 2 & 2 & 11371.637 & -0.002 \\ 2 & 4 & 2 & 4 & 11372.365 & -0.003 \\ 2 & 3 & 0 & 3 & 11372.424 & -0.001 \\ 2 & 5 & 2 & 5 & 11373.055 & -0.001 \\ 1 & 3 & 1 & 3 & 11373.198 & -0.002\end{array}$

Table S2. Observed frequencies and residuals (in $\mathrm{MHz}$ ) for the nuclear quadrupole coupling hyperfine components of the ${ }^{15} \mathrm{~N}(1)$ and ${ }^{15} \mathrm{~N}(3)$ isotopically substituted uracil:

\begin{tabular}{|c|c|c|c|c|c|c|c|c|c|c|c|}
\hline \multirow[b]{2}{*}{$J^{\prime}$} & \multirow[b]{2}{*}{$K_{-1}^{\prime}$} & \multirow[b]{2}{*}{$K_{+1}^{\prime}$} & \multirow[b]{2}{*}{$J^{\prime \prime}$} & \multirow[b]{2}{*}{$K_{-1}^{\prime \prime}$} & \multirow[b]{2}{*}{$K_{+1}^{\prime \prime}$} & \multirow[b]{2}{*}{$F^{\prime}$} & \multicolumn{3}{|c|}{${ }^{15} \mathrm{~N}_{1}{ }^{14} \mathrm{~N}_{3}$} & \multicolumn{2}{|c|}{${ }^{14} \mathrm{~N}_{1}-{ }^{15} \mathrm{~N}_{3}$} \\
\hline & & & & & & & $F^{\prime \prime}$ & Obs / MHz & Obs-cal / MHz & Obs / MHz & $\begin{array}{c}\text { Obs-cal / } \\
\mathrm{MHz}\end{array}$ \\
\hline \multirow[t]{3}{*}{1} & 1 & 1 & 0 & 0 & 0 & 2 & 1 & 5176.563 & 0.002 & 5184.802 & 0.001 \\
\hline & & & & & & 1 & 1 & 5177.013 & -0.001 & 5185.398 & 0.002 \\
\hline & & & & & & 0 & 1 & 5175.875 & -0.004 & 5183.910 & 0.001 \\
\hline \multirow[t]{2}{*}{2} & 1 & 2 & 1 & 0 & 1 & 3 & 2 & 7821.700 & 0.003 & 7840.335 & 0.000 \\
\hline & & & & & & 2 & 1 & 7822.222 & -0.003 & 7841.012 & 0.002 \\
\hline \multirow[t]{3}{*}{2} & 2 & 0 & 1 & 1 & 1 & 3 & 2 & 13735.298 & 0.000 & 13758.853 & 0.001 \\
\hline & & & & & & 2 & 1 & 13734.005 & 0.001 & 13757.429 & 0.000 \\
\hline & & & & & & 1 & 0 & 13736.446 & 0.001 & 13760.204 & 0.000 \\
\hline \multirow[t]{2}{*}{2} & 2 & 1 & 1 & 1 & 0 & 3 & 2 & 12884.660 & 0.003 & 12899.020 & -0.002 \\
\hline & & & & & & 2 & 1 & 12885.065 & -0.005 & 12899.578 & -0.001 \\
\hline \multirow[t]{2}{*}{3} & 1 & 3 & 2 & 0 & 2 & 4 & 3 & 10191.430 & 0.000 & 10218.903 & 0.000 \\
\hline & & & & & & 3 & 2 & 10191.963 & 0.003 & 10219.544 & 0.000 \\
\hline \multirow[t]{3}{*}{4} & 0 & 4 & 3 & 1 & 3 & 5 & 4 & 11306.228 & -0.001 & 11363.676 & -0.002 \\
\hline & & & & & & 4 & 3 & 11306.287 & -0.001 & 11363.720 & 0.000 \\
\hline & & & & & & 3 & 2 & 11306.342 & 0.000 & 11363.804 & 0.001 \\
\hline
\end{tabular}

Table S3. Observed frequencies and residuals (in MHz) for the rotational transitions of the ${ }^{15} \mathrm{~N}_{1}{ }^{-{ }^{15}} \mathrm{~N}_{3},{ }^{15} \mathrm{~N}_{1}-{ }^{15} \mathrm{~N}_{3}-{ }^{13} \mathrm{C}_{2}$ and ${ }^{15} \mathrm{~N}_{1}$ ${ }^{15} \mathrm{~N}_{3-}{ }^{13} \mathrm{C}_{4}$ isotopically substituted uracil:

\begin{tabular}{|c|c|c|c|c|c|c|c|c|c|c|c|}
\hline \multirow[b]{2}{*}{$J^{\prime}$} & \multirow[b]{2}{*}{$K_{-1}^{\prime}$} & \multirow[b]{2}{*}{$K_{+1}^{\prime}$} & \multirow[b]{2}{*}{$J^{\prime \prime}$} & \multirow[b]{2}{*}{$K_{-1}^{\prime \prime}$} & \multirow[b]{2}{*}{$K_{+1}^{\prime \prime}$} & \multicolumn{2}{|c|}{${ }^{15} \mathrm{~N}_{1}-{ }^{15} \mathrm{~N}_{3}$} & \multicolumn{2}{|c|}{${ }^{15} \mathrm{~N}_{1}-{ }^{15} \mathrm{~N}_{3}-{ }^{13} \mathrm{C}_{2}$} & \multicolumn{2}{|c|}{${ }^{15} \mathrm{~N}_{1^{-}}{ }^{15} \mathrm{~N}_{3^{-}}{ }^{13} \mathrm{C}_{4}$} \\
\hline & & & & & & Obs / MHz & Obs-cal / MHz & Obs / MHz & Obs-cal / MHz & Obs / MHz & Obs-cal / MHz \\
\hline 1 & 1 & 1 & 0 & 0 & 0 & 5146.656 & 0.001 & 5137.289 & 0.001 & 5138.198 & 0.000 \\
\hline 2 & 1 & 2 & 1 & 0 & 1 & 7785.579 & 0.001 & 7765.468 & 0.000 & 7765.132 & -0.001 \\
\hline 2 & 2 & 0 & 1 & 1 & 1 & 13657.869 & 0.001 & 13631.419 & 0.001 & 13633.390 & 0.001 \\
\hline 2 & 2 & 1 & 1 & 1 & 0 & 12801.035 & -0.002 & 12783.679 & -0.002 & 12787.654 & -0.001 \\
\hline 3 & 1 & 3 & 2 & 0 & 2 & 10148.977 & 0.001 & 10119.708 & 0.001 & 10118.396 & 0.001 \\
\hline 4 & 0 & 4 & 3 & 1 & 3 & 11298.342 & -0.001 & 11239.934 & -0.001 & 11231.021 & 0.000 \\
\hline
\end{tabular}


Table S4. Observed frequencies and residuals (in MHz) for the rotational transitions of the ${ }^{15} \mathrm{~N}_{1^{-}}{ }^{15} \mathrm{~N}_{3^{-}}{ }^{13} \mathrm{C}_{5}$ and ${ }^{15} \mathrm{~N}_{1^{-}}{ }^{15} \mathrm{~N}_{3}-{ }^{13} \mathrm{C}_{6}$ isotopically substituted uracil:

\begin{tabular}{|c|c|c|c|c|c|c|c|c|c|}
\hline \multirow[b]{2}{*}{$J^{\prime}$} & \multirow[b]{2}{*}{$K_{-1}^{\prime}$} & \multirow[b]{2}{*}{$K_{+1}^{\prime}$} & \multirow[b]{2}{*}{$J^{\prime \prime}$} & \multirow[b]{2}{*}{$K_{-1}^{\prime \prime}$} & \multirow[b]{2}{*}{$K_{+1}^{\prime \prime}$} & \multicolumn{2}{|c|}{${ }^{15} \mathrm{~N}_{1}{ }^{15} \mathrm{~N}_{3^{-}}{ }^{13} \mathrm{C}_{5}$} & \multicolumn{2}{|c|}{${ }^{15} \mathrm{~N}_{1^{-}}{ }^{15} \mathrm{~N}_{3^{-}}{ }^{13} \mathrm{C}_{6}$} \\
\hline & & & & & & Obs / MHz & Obs-cal / MHz & Obs / MHz & Obs-cal / MHz \\
\hline 1 & 1 & 1 & 0 & 0 & 0 & 5099.704 & 0.000 & 5051.099 & -0.001 \\
\hline 2 & 1 & 2 & 1 & 0 & 1 & 7719.790 & 0.000 & 7669.464 & 0.000 \\
\hline 2 & 2 & 0 & 1 & 1 & 1 & 13534.754 & 0.000 & 13413.108 & 0.000 \\
\hline 2 & 2 & 1 & 1 & 1 & 0 & 12679.020 & -0.001 & 12534.931 & 0.000 \\
\hline 3 & 1 & 3 & 2 & 0 & 2 & 10065.915 & 0.000 & 10012.363 & 0.001 \\
\hline 4 & 0 & 4 & 3 & 1 & 3 & 11228.562 & 0.000 & 11268.368 & 0.000 \\
\hline
\end{tabular}

Table S5. Observed frequencies and residuals (in MHz) for the rotational transitions of the ${ }^{15} \mathrm{~N}_{1^{-}}{ }^{15} \mathrm{~N}_{3^{-}}{ }^{18} \mathrm{O}_{7}$ and ${ }^{15} \mathrm{~N}_{1^{-}}{ }^{15} \mathrm{~N}_{3-}{ }^{18} \mathrm{O}_{8}$ isotopically substituted uracil:

\begin{tabular}{|c|c|c|c|c|c|c|c|c|c|}
\hline \multirow[b]{2}{*}{$J^{\prime}$} & \multirow[b]{2}{*}{$K_{-1}^{\prime}$} & \multirow[b]{2}{*}{$K_{+1}^{\prime}$} & \multirow[b]{2}{*}{$J^{\prime \prime}$} & \multirow[b]{2}{*}{$K_{-1}^{\prime \prime}$} & \multicolumn{3}{|c|}{${ }^{15} \mathrm{~N}_{1^{-}}{ }^{15} \mathrm{~N}_{3^{-}}{ }^{18} \mathrm{O}_{7}$} & \multicolumn{2}{|c|}{${ }^{15} \mathrm{~N}_{1}{ }^{15} \mathrm{~N}_{3^{-}}{ }^{18} \mathrm{O}_{8}$} \\
\hline & & & & & $K_{+1}^{\prime \prime}$ & Obs / MHz & Obs-cal / MHz & Obs / MHz & Obs-cal / MHz \\
\hline 1 & 1 & 1 & 0 & 0 & 0 & 5053.130 & 0.002 & 5059.229 & 0.002 \\
\hline 2 & 1 & 2 & 1 & 0 & 1 & 7612.797 & 0.003 & 7615.268 & 0.002 \\
\hline 2 & 2 & 0 & 1 & 1 & 1 & 13402.177 & 0.002 & 13417.007 & 0.001 \\
\hline 2 & 2 & 1 & 1 & 1 & 0 & 12599.715 & -0.004 & 12621.640 & -0.002 \\
\hline 3 & 1 & 3 & 2 & 0 & 2 & 9907.756 & 0.000 & 9907.561 & 0.000 \\
\hline 4 & 0 & 4 & 3 & 1 & 3 & 10892.528 & -0.001 & 10862.284 & -0.001 \\
\hline
\end{tabular}

Table S6. Atom coordinates in the principal inertial axis system of uracil.

\begin{tabular}{l|ccc|c}
\hline & $\mathrm{a}$ & $\mathrm{b}$ & $\mathrm{c}^{\mathrm{b}}$ & \\
\hline $\mathrm{N}_{1}$ & $1.1884(10)^{\mathrm{a}}$ & $-1.0115(12)$ & 0 & \\
$\mathrm{C}_{2}$ & $1.1975(10)$ & $0.375(3)$ & 0 & \\
$\mathrm{~N}_{3}$ & $-0.091(13)$ & $0.9640(12)$ & 0 & \\
$\mathrm{C}_{4}$ & $-1.2925(9)$ & $0.295(4)$ & 0 & \\
$\mathrm{C}_{5}$ & $-1.1992(10)$ & $-1.1535(10)$ & 0 & \\
$\mathrm{C}_{6}$ & $0.05(2)$ & $-1.7396(7)$ & 0 & \\
$\mathrm{O}_{7}$ & $2.2323(5)$ & $1.0189(12)$ & 0 & \\
$\mathrm{O}_{8}$ & $-2.3273(5)$ & $0.9432(13)$ & 0 & \\
\hline
\end{tabular}

\footnotetext{
${ }^{a}$ Derived errors in parentheses in units of the last digit, calculated according to Costain's formula (J.Chem.Phys. 1958, 28, 864): $\delta z_{i}=0.0012 /\left|z_{i}\right|{ }^{b}$ Assumed value for all atoms.
} 
Complete reference [20]:

Gaussian 03, Revision B.04,

M. J. Frisch, G. W. Trucks, H. B. Schlegel, G. E. Scuseria, M. A. Robb, J. R. Cheeseman, J. A. Montgomery, Jr., T. Vreven, K. N. Kudin, J. C. Burant, J. M. Millam, S. S. Iyengar, J. Tomasi, V. Barone, B. Mennucci, M. Cossi, G. Scalmani, N. Rega, G. A. Petersson, H. Nakatsuji, M. Hada, M. Ehara, K. Toyota, R. Fukuda, J. Hasegawa, M. Ishida, T. Nakajima, Y. Honda, O. Kitao, H. Nakai, M. Klene, X. Li, J. E. Knox, H. P. Hratchian, J. B. Cross, C. Adamo, J. Jaramillo, R. Gomperts, R. E. Stratmann, O. Yazyev, A. J. Austin, R. Cammi, C. Pomelli, J. W. Ochterski, P. Y. Ayala, K. Morokuma, G. A. Voth, P. Salvador, J. J. Dannenberg, V. G. Zakrzewski, S. Dapprich, A. D. Daniels, M. C. Strain, O. Farkas, D. K. Malick, A. D. Rabuck, K. Raghavachari, J. B. Foresman, J. V. Ortiz, Q. Cui, A. G. Baboul, S. Clifford, J. Cioslowski, B. B. Stefanov, G. Liu, A. Liashenko, P. Piskorz, I. Komaromi, R. L. Martin, D. J. Fox, T. Keith, M. A. Al-Laham, C. Y. Peng, A. Nanayakkara, M. Challacombe, P. M. W. Gill, B. Johnson, W. Chen, M. W. Wong, C. Gonzalez, and J. A. Pople, Gaussian, Inc., Pittsburgh PA, 2003. 